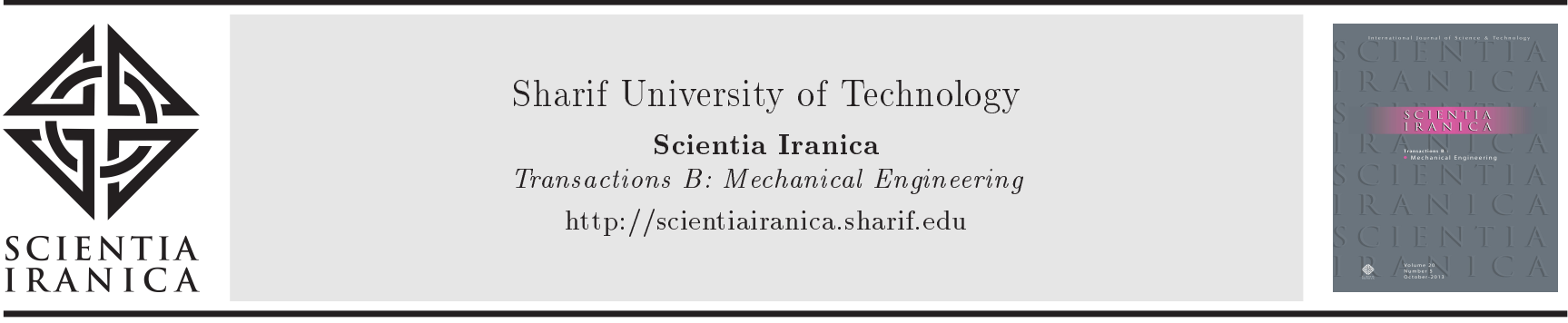

\title{
Using an optimized RBF neural network to predict the out-of-plane welding distortions based on the 3-2-1 locating scheme
}

\author{
M.M. Tafarroj and F. Kolahan* \\ Department of Mechanical Engineering, Ferdowsi University of Mashhad, Mashhad, Iran. \\ Received 28 October 2017; received in revised form 12 December 2017; accepted 8 January 2018
}

\author{
KEYWORDS \\ GTAW; \\ Distortion; \\ 3-2-1 locating scheme; \\ $\mathrm{RBF}$; \\ Optimization; \\ Simulated annealing.
}

\begin{abstract}
This study deals with the effect of locator positioning in the 3-2-1 locating scheme to control the out-of-plane distortion in gas tungsten arc welding of sheet metals. To apply this locating scheme to the sheet metals, a suitable fixture was designed. The distortion of the welded plates was predicted using the Radial Basis Function (RBF) neural network. To gather the experimental data employed in the RBF modeling process, a set of welding tests was performed on the sheet specimens by varying the positions of the three locators. The parameters of the network were optimally selected using the Simulated Annealing (SA) optimization algorithm. The average and maximum errors computed for the test dataset were respectively $2.43 \%$ and $5.30 \%$ while in some cases, the error fell below $1 \%$. The results of the RBF network showed very good agreement with the experiments and it can be concluded that this modeling technique can be utilized successfully in predicting the welding distortions when the 3-2-1 locating scheme is used.
\end{abstract}

(C) 2019 Sharif University of Technology. All rights reserved.

\section{Introduction}

Welding is one of the most well-known methods to join materials permanently. Roughly speaking, welding of machinery parts is inevitable for most of the engineering requests [1]. Selecting the welding process type is contingent on the structure, technical requirements, and application conditions [2]. As a class of fusion welds, the Gas Tungsten Arc Welding (GTAW), also known as the Tungsten Inert Gas (TIG) welding process, is widely used for joining thin sheet metals remarkably implemented in aerospace, naval, and automobile industries. This type of welds is performed by the use of a non-consumable tungsten electrode together with inert shielding gas, like argon, which

\footnotetext{
*. Corresponding author.

E-mail address: kolahan@um.ac.ir (F. Kolahan)
}

protects the weld from atmospheric contamination [3]. However, extremely non-uniform heating during the welding process causes distortion, especially in thin structures, since the weldment heats and cools. The welding distortions will lead to many problems like loss of dimensional control and structural integrity, difficulty in subsequent alignment with the adjacent component, and increase in fabrication costs with straightening [4].

As shown in Figure 1, welding distortions can be classified into six different types including transverse shrinkage, longitudinal shrinkage, and rotational distortion, which are known as the in-plane distortions, and the angular distortion, bending distortion, and buckling, which are the out-of-plane distortions [4-6]. It should be noted that multiple types of these distortions may occur at the same time in welded structures and it is sometimes difficult to distinguish the types of distortion present in a workpiece [5].

Many factors affect the welding induced distor- 


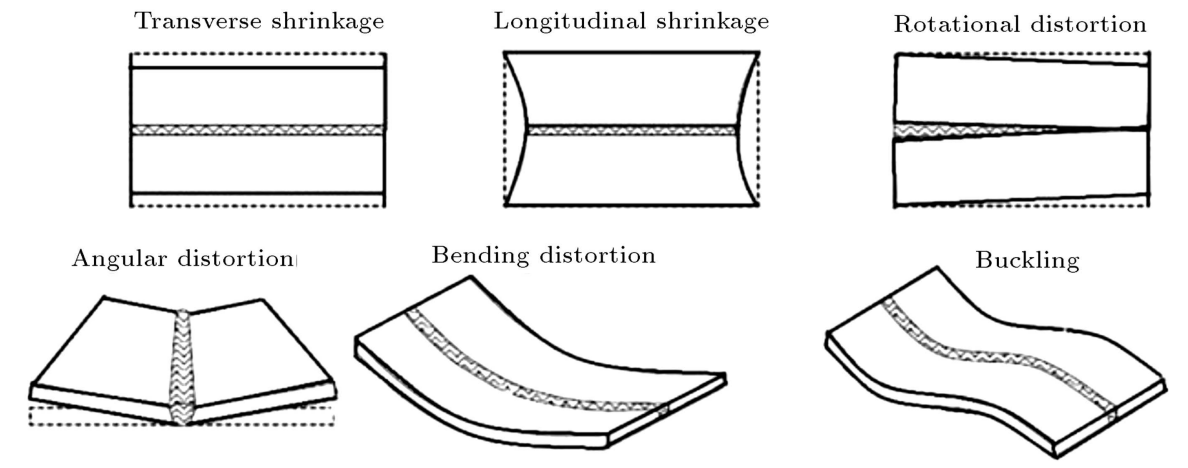

Figure 1. Various types of welding distortions [5].

tions. Thus, it is difficult to control this phenomenon by only relying on practical experiences. If distortions can be forecasted before welding, mitigating of this unwanted event can be done successfully [7].

Many studies have been done to predict and control the welding induced distortions. Thanks to the development of high-speed computers, the Finite Element Analysis (FEA) can be used to simulate and predict the welding distortions. Pazooki et al. [5] conducted a study to control the gas metal arc welding distortions by the use of additional heat source. In addition to the experiments, they used a $3 \mathrm{D}$ finite element model to understand the distortion reduction mechanism. Deng et al. [7] investigated the effect of external restraint on the welding deformations. In their research, six clamps were employed to restrain the welded specimens. Both numerical results and experiments proved that external restraint could mitigate the final deformation to some extent. Li et al. [8] investigated the effect of welding parameters on the weld shape and distortion of the $\mathrm{Ti} 2 \mathrm{AlNb}$ alloy joint. The effect of fixtures on the welding distortion was also studied, and it was shown that welding without fixtures in bead-on-plate welding would cause less distortion than the butt welds.

Compared to their many capabilities in modeling of various processes, the applications of Artificial Neural Networks (ANNs) in predicting the welding induced distortion are still rare. Therefore, the implementation of ANNs and meta-heuristic optimization algorithms is a promising and exciting area of welding researches. Lightfoot et al. [9] established an ANN model to predict the welding distortion of the steel plates. They also performed a sensitivity analysis to find out the factors affecting the welding distortion. Bruce and Lightfoot [10] modeled the distortion caused by welding operation using the ANN and found that the results of the ANN well fitted the actual values. Yang and Shao [11] developed a thermo-mechanical model to forecast the welding residual stress and distortion. In this research, the welding sequence causing the minimum distortion was determined using the Genetic Algorithm (GA), and at last, the optimization strategy was successfully pursued for a real product. Choobi et al. [12] used an ANN structure to forecast the angular distortions in single-pass butt-welded 304 stainless steel plates. They employed a set of FEM results obtained for various plate dimensions as the ANN inputs. Barclay et al. [13] investigated the suitability of the induction heating process with the traveling induction coil to rectify the welding angular distortion. By using the experimental data, they considered an ANN to predict the distortion. According to their findings, the ANN was well-suited to predict the final distortion of the plate for both after-welding and induction heating. Tian et al. [14] developed a backpropagation neural network to predict the transverse shrinkage and angular distortion in bead-on-plate GTA welding of S304L stainless steel. The employed data to construct the neural network were captured from the FEM simulation while the accuracy of numerical results was verified by the experiments. The same work has also been performed by Narayanareddy et al. [15].

As it can be seen, several studies have been conducted on the welding distortions. However, the work on the effect of jigs and fixtures on the welding induced distortions is relatively rare $[7,8,16-18]$. Therefore, the effect of jigs and fixture as the external mechanical constraints affecting the welding distortions can be further attended to. In addition, a thorough search through the relevant literature on the application of 32-1 locating scheme in arc welding operations yielded very limited works [19].

This paper focuses on the application of 3-2-1 locating scheme in GTAW process for sheet metals with regards to the resultant distortion in the workpiece. The main purpose is to evaluate the out-of-plane distortion, as affected by various arrangements of locators. To achieve this, an Artificial Neural Network (ANN) model is developed for accurately predicting the final distortion of the welded workpiece when the positions of locators are simultaneously changed.

The study is organized as follows: in Section 2, the 3-2-1 locating scheme is introduced. In Section 3, the tools used in this work, the Radial Basis Function (RBF) neural network, and the Simulated Annealing 
(SA) optimization technique are briefly presented. Section 4 explains the procedure for the experimental work, including experiment setups and output measurements. The main findings of this research are presented and discussed in Section 5. Finally, the concluding remarks are rendered in Section 6 .

\section{The 3-2-1 locating scheme}

The 3-2-1 locating scheme is a method utilized to restrain all 6 (Degree of Freedom) $\mathrm{DoFs}^{2}$ of a workpiece. According to Figure 2, the points A1, A2, and A3 construct a plane and restrain the translation in $z$ direction and rotation in $x$ - and $y$-directions. Additionally, the points B1 and B2 define a line and restrain the translation in $y$-direction and rotation in $z$-direction. Finally, C1 locks the translation in $x$-direction. An adequately designed fixture for sheet metals usually needs $N \geq 3$ carefully selected locators on the primary datum plane to restrain deformation of the part [20].

In 3-2-1 locating approach, the 2-1 locating is applied by using a 4 -way pin and a 2 -way pin. A typical illustration of the 3-2-1 locating scheme is provided in Figure 3. As can be seen, the 4-way and 2-way pins restrain the translations in $x$ - and $y$-directions and

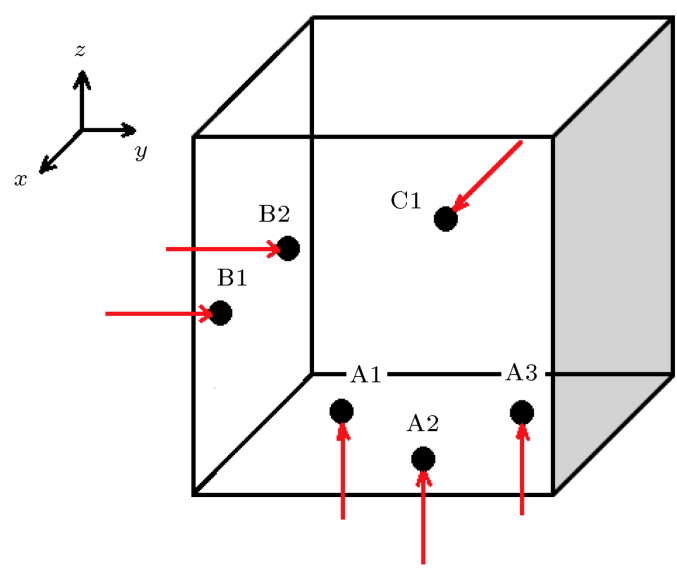

Figure 2. The 3-2-1 locating scheme.

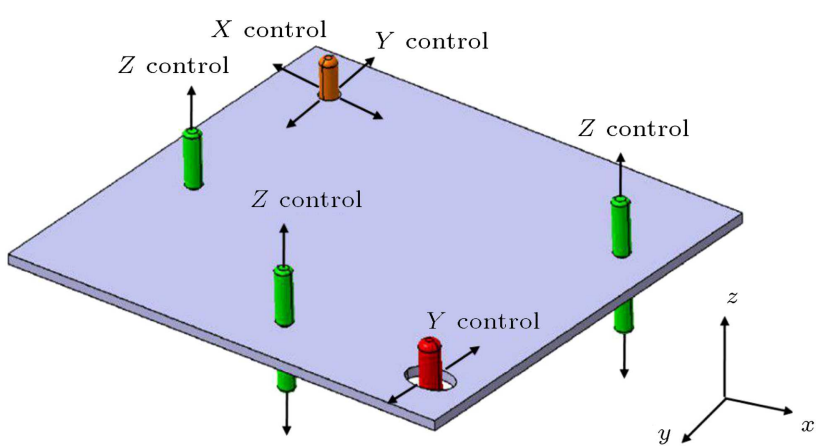

Figure 3. A typical 3-2-1 locating scheme applied to a plate. rotation in $z$-direction.

For any arrangement of locators constraining the $z$-direction and the rotations in $x$ - and $y$-directions, if a method is presented to predict the distortion prior to the welding operation, considerable values of time and cost can be saved.

\section{Modeling approach}

\subsection{The Radial Basis Function (RBF) network}

An artificial neural network is a processing system of information initially inspired by the biological neural units. ANNs method can be a noteworthy tool for material design. It is common to regard an ANN as a "black box" concentrated on the prediction accuracy [21].

The radial basis functions are neural networks with three fixed layers. Simple structure of such networks has the advantage of lower processing time. The ability of online learning, high power of extending the results and generalization, and high tolerance to input noises are the other advantages of the RBF networks [22]. Thanks to the high capability of generalization, the RBFs are suitable techniques to figure out the patterns not used in training procedure. RBF networks have robust tolerance to the input noise, which improves the stability of the constituted systems. Therefore, it is sensible to consider RBF as a competitive approach to modeling of nonlinear problems [23]. As stated previously, an RBF has three layers. The numbers of input and output neurons are imposed by the problem at hand. The Gaussian functions are implemented in the hidden layer while the sigmoid or linear functions are used in the output layer. A schematic view of the constructed RBF is illustrated in Figure 4. In the figure, inputs are the positions of the locators represented by $P_{i}$ and the output is the welding induced distortion.

In the RBF, the goal is to define a function like $f(\vec{X})$ shown in Eq. (1).

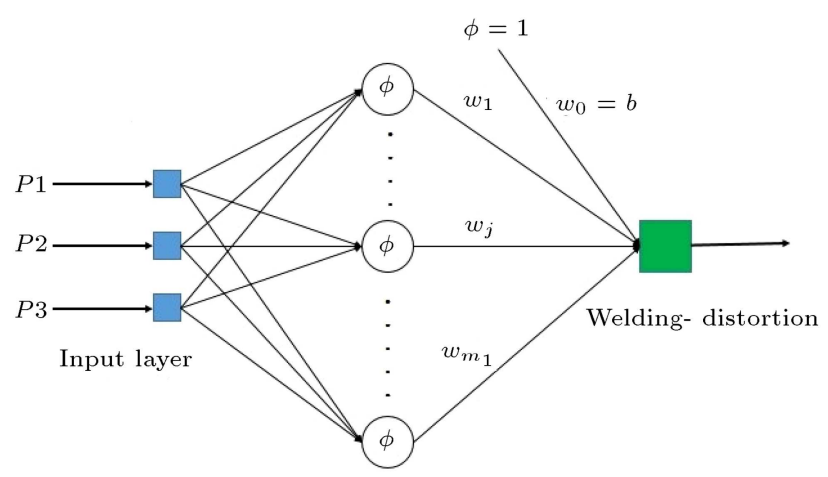

Hidden layer of $m_{1}$ basis functions

Figure 4. Schematic view of the RBF neural network. 


$$
f\left(\vec{X}^{p}\right)=t^{p} \quad \forall p=1,2, \ldots, P
$$

where $\vec{X}^{p}$ is the pattern number $p$ and $t^{p}$ is its corresponding desired value. This can be done by mapping the $D$-dimensional input vectors $\vec{X}^{p}=\left\{X_{i}^{p}\right.$ : $i=1, \ldots, D\}$ onto the output $t^{p}$. The RBF defines $m_{1} \leq$ $P$ basis functions based on the form $\phi\left(\left\|\vec{X}-\vec{X}^{p}\right\|\right)$ where $\phi$ is a nonlinear function. Therefore, the output function can be generally formulated via Eq. (2).

$$
f(\vec{X})=\sum_{p=1}^{P} w_{p} \phi\left(\left\|\vec{X}-\vec{X}^{p}\right\|\right) .
$$

Now, the problem is to find the weights $w_{p}$ such that the function can be fitted to the data. Combining both Eqs. (1) and (2) yields Eq. (3), which is useful to evaluate the weights:

$$
f\left(\vec{X}^{q}\right)=\sum_{p=1}^{P} w_{p} \phi\left(\left\|\vec{X}^{q}-\vec{X}^{p}\right\|\right)=t^{q} .
$$

Introducing the matrix notations $\mathbf{t}=\left\{t^{p}\right\}, \mathbf{w}=\left\{w_{p}\right\}$, and $\boldsymbol{\Phi}=\left\{\boldsymbol{\Phi}_{p q}=\phi\left(\left\|\vec{X}^{q}-\vec{X}^{p}\right\|\right)\right.$, Eq. (3) can be written as Eq. (4):

$$
\Phi \mathbf{w}=\mathbf{t}
$$

Now, having the inverse of matrix $\boldsymbol{\Phi}$, one can simply attain the weight matrix via Eq. (5).

$$
\boldsymbol{w}=\boldsymbol{\Phi}^{-\mathbf{1}} \mathbf{t}
$$

When the weights are available, a function $f(\vec{X})$ exists that can be fitted to the point data. The most common basis function is Gaussian function (Eq. (6)):

$$
\phi(r)=\exp \left(-\frac{r^{2}}{2 \sigma^{2}}\right)(\sigma>0) .
$$

In Eq. (6), the value of $\sigma$ is known as the spread. To achieve the best performance of the RBF network, this parameter together with the maximum number of neurons added to the hidden layer must be carefully determined. Thus, an optimization procedure using the Simulated Annealing (SA) algorithm is utilized in the following for optimal determining of these parameters.

\subsection{RBF optimization procedure}

The RBF is employed to predict the welding induced distortion. To get the best performance of the RBF, its parameters should be optimally selected. Therefore, the simulated annealing optimization algorithm is employed to determine the best combination for the values of the RBF parameters.

Determining the best combination of variables which yields the best process performance is the goal of an optimization process [24]. Simulated annealing is an optimization algorithm first introduced by Kirkpatrick et al. to solve hard optimization problems [25]. It was inspired by the process of physical annealing of solids.

In the annealing process, a solid is heated to a high temperature until all molecules can move freely. Annealing (slow cooling) would result in a perfect crystalline in which all atoms are arranged in low-level energy lattice.

In the SA algorithm, slow cooling of the metallurgical annealing is simulated by the parameter $T$ and the rate of its reduction (called cooling schedule). Starting from a high temperature and slowly reducing it, to some extent, prevents being trapped in local optima.

Therefore, in early iterations, the value of $T$ should be high enough to make sure of escaping from local optima. In the beginning, when $T$ is high, nonimproving solutions are more likely to be accepted. When the algorithm proceeds, the value of temperature is gradually reduced and, hence, only the improving solutions are accepted.

The main idea of this technique is to generate a random solution. Then, a new solution is created in the neighborhood of the current solution. If the new point is better, it is accepted; but if it is worse, the new solution can be accepted or rejected based on probability. For minimizing an objective, the pseudocode of the SA algorithm can be written as:

1. Select a random initial point $\vec{X}_{0}$, call the RBF, and evaluate the objective function $f\left(\vec{X}_{0}\right)$;

2. Generate a new point $\vec{X}$ in the neighborhood of $\vec{X}_{0}$, call the RBF, and evaluate the objective function $f(\vec{X})$;

3. Calculate $\Delta f=f(\vec{X})-f\left(\vec{X}_{0}\right)$ and $p=e^{\frac{-\Delta f}{T}}$;

4. If $\Delta f<0$, set $\vec{X}_{0}=\vec{X}$ and go to step 5. Otherwise, generate a random number $\beta \in\left(\begin{array}{ll}0 & 1\end{array}\right)$; if $\beta<p$, set $\vec{X}_{0}=\vec{X}$ and go to step 5 . Else, go to step 2 ;

5. Reduce the value of $T$ by some linear or logarithmic approach (for example, $T=k T$ where $0<k<1$ );

6 . If the stopping criterion of the algorithm is not satisfied, go to step 2 as the next iteration; otherwise, go to step 6;

7. Stop the algorithm and present the optimal point.

At each iteration, to generate a new solution vector $(\vec{X})$ in step 2 of the SA algorithm, an element of the current solution vector $\left(\vec{X}_{0}\right)$ is selected randomly. Then, the element is changed to a random value within its permitted range. For example, assume that $X_{0}=(0.11,4)$ and the first element of $\vec{X}_{0}$ (i.e. 0.11 ) is randomly selected to change. It is supposed that the permitted range of the selected element is $[0.1,10]$. Therefore, a random value is generated in this range 
(e.g. 0.80). Now, the new generated neighbor solution to be evaluated is $\vec{X}=(0.80,4)$.

The two main parameters of the RBF which should be optimally tuned are the spread $(S p)$ and the maximum number of neurons $(N)$. The objective function of the problem can be stated by Eq. (7):

$$
\begin{aligned}
& \min f(\vec{X})=M R E(\vec{X})+a b s(1 / C r) \\
& 0.1 \leq S p \leq 10 \\
& 1 \leq N \leq 15
\end{aligned}
$$

In Eq. $(7), \vec{X}=(S p, N)$ is the solution vector containing the optimization variables, the $M R E$ is the mean relative error computed for test dataset, and $\mathrm{Cr}$ is the correlation coefficient between the target values (values obtained from the experiments) and the network outputs computed for the test dataset. After the process of determining the optimum values of the RBF parameters, the results of the optimized RBF will be presented.

\section{Experimental procedure}

\subsection{Materials and equipment}

An air-cooled PSQ 250 AC/DC (GAAM ELECTRICCo, Iran) semi-automatic welding machine with 250 ampere capacity and high pulse frequency (up to $500 \mathrm{~Hz}$ ) was used to perform experiments on the St12 steel sheets. The relative motion between the weldments and the torch was carried out using an automatic table with controllable linear motion. A new fixture was designed, which was suitably able to apply the N-2-1 locating scheme. When the plate is placed between the upper and lower parts of the fixture, the locators fix the plate in $z$-direction, which is normal to the plate, and avoid the rotation in $x$ - and $y$-directions. The welding machine, automated table, the GTAW air-cooled torch with ceramic nozzle, and the designed fixture are exhibited in Figure 5.

Because of good welding performance and relatively high dimensional accuracy, the St12 steel has widely been employed to manufacture various automotive parts. The chemical composition and mechanical properties of St12 steels are respectively presented in Tables 1 and 2. The shielding gas was pure argon and because the plate was thin, the welding was done without any filler metal.

Table 1. Chemical composition of St12 steel (wt\%).

\begin{tabular}{ccccc}
\hline Element & $\mathbf{C}$ & $\mathbf{M n}$ & $\mathbf{P}$ & $\mathbf{S}$ \\
\hline Amount & 0.12 & 0.6 & 0.045 & 0.04 \\
\hline
\end{tabular}
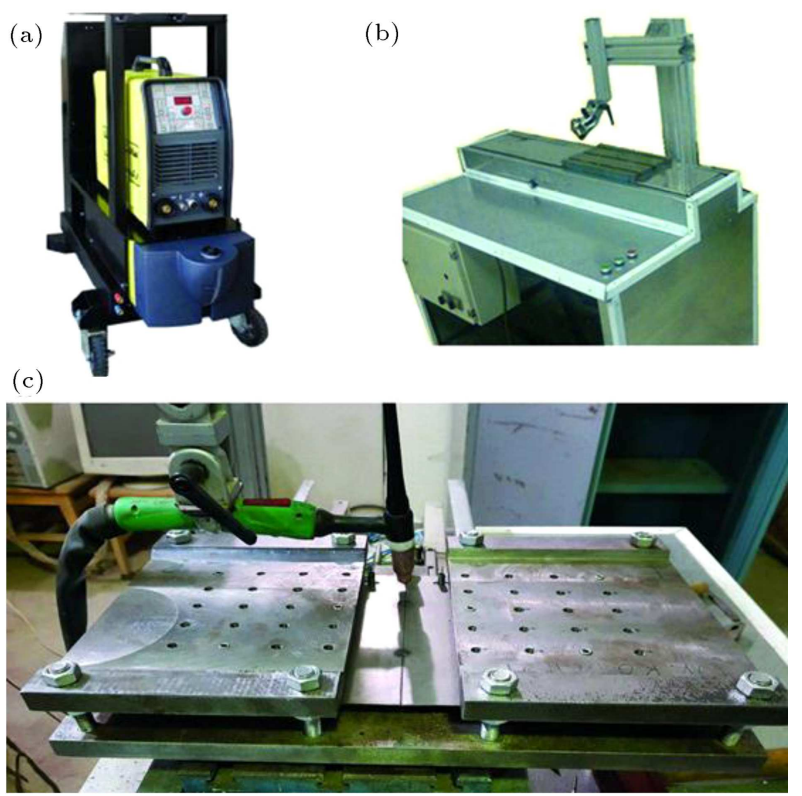

Figure 5. The equipment used in experiments: (a) The PSQ 250 AC/DC welding machine, (b) the table with controllable linear motion, and (c) the welding torch and the designed fixture.

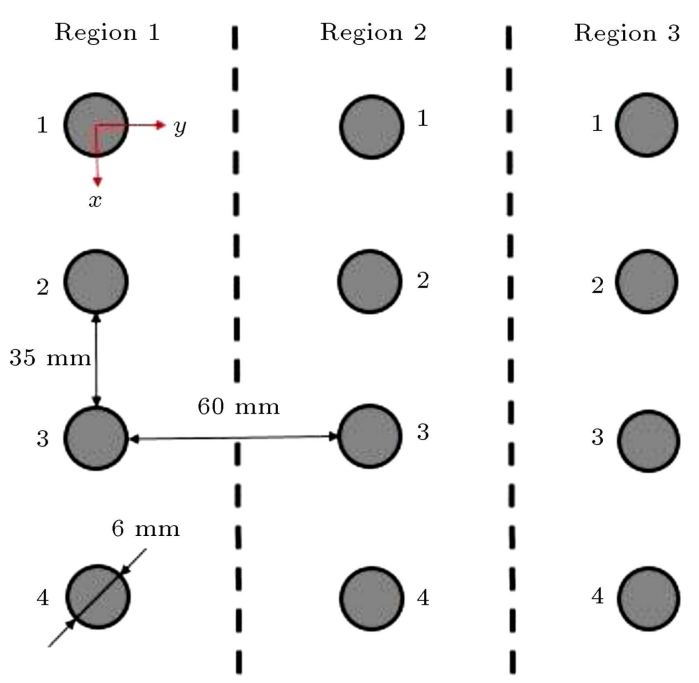

Figure 6. The predetermined sub-regions in which the locators could move.

Table 2. Mechanical properties of St12 steel.

\begin{tabular}{cccc}
\hline Property & $\begin{array}{c}\text { Tensile } \\
\text { strength }\end{array}$ & $\begin{array}{c}\text { Yield } \\
\text { strength }\end{array}$ & $\begin{array}{c}\text { Total } \\
\text { elongation }\end{array}$ \\
\hline Value & $270-410 \mathrm{MPa}$ & $140-280 \mathrm{MPa}$ & $\geq 28 \%$ \\
\hline
\end{tabular}

\subsection{Welding experiments and measurements}

After setting up the required equipment, the specimens were welded together. Since the independent variables were the locations of three locators, as can be seen in Figure 6, the plate region was divided into three sub-regions and the locators could be moved to some 
Table 3. The values of welding parameters.

\begin{tabular}{cccccc}
\hline Parameter & Current (I) & Voltage (V) & Speed (S) & Torch height (h) & Gas flow rate (Q) \\
\hline Value & $100 \mathrm{~A}$ & $14 \mathrm{~V}$ & $160 \mathrm{~mm} / \mathrm{min}$ & $2 \mathrm{~mm}$ & $8 \mathrm{~L} / \mathrm{min}$ \\
\hline
\end{tabular}

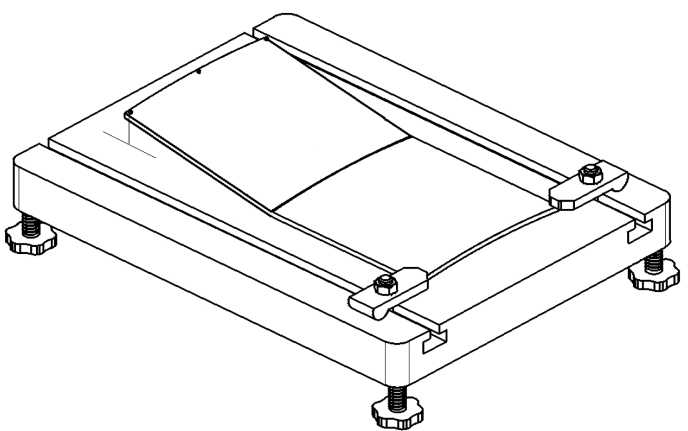

Figure 7. The method of distortion measuring.

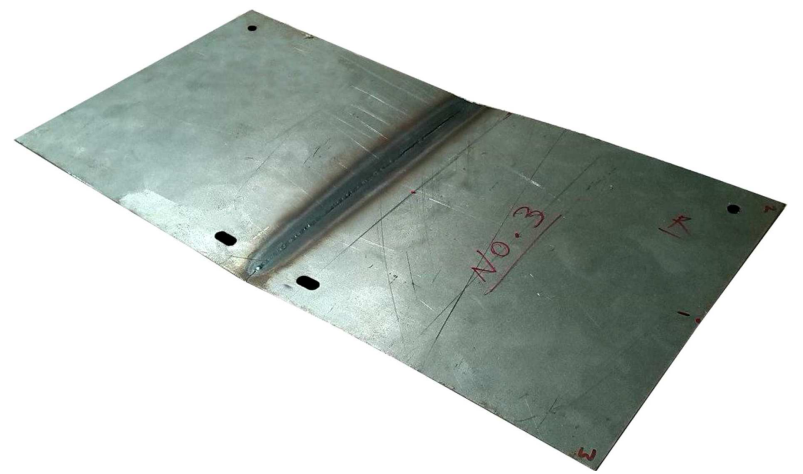

Figure 8. A sample of the welded plates.

predetermined locations.Thus, as can be seen in Figure 6 , each design variable had four levels.

The other welding parameters (current, voltage, speed, gas flow rate, torch height with respect to the plate surface) shown in Table 3 were constant during the welding operation.

Before welding, three points on the plate were marked to measure the welding distortion. After welding, the displacement of the three points with respect to the surface underneath was measured using a simple method, which is illustrated in Figure 7. Then, the obtained values were averaged and the distortion value of the welded plate was recorded.

Based on the design assumption, 21 experiments were conducted. The locators' $(x, y)$ coordinates along with their resultant distortion are reported in Table 4. A sample of the welded plates is demonstrated in Figure 8.

The procedure for conducting the welding experiments, when the 3-2-1 locating scheme is applied to control the welding distortion, is very time consuming and expensive. Thus, it is necessary to introduce a novel approach to predict the welding induced distortion when the 3-2-1 locating scheme is applied. In the next section, an RBF artificial neural network is constructed to solve the problem.

\section{Results and discussion}

The experimental data were employed to train an RBF model. For this purpose, each predetermined position of locators shown in Figure 6 was coded by a number. For instance, in region 1 , the first, second, third, and fourth locations were respectively coded by $1,2,3$, and 4. This coding method was also used for the other two regions. With this coding method, the first experiment had the inputs $(1,1,1)$ and so on. Among all data reported in Table 4, six data were set off from the rest as the test data, which were not used in the training process. These data were presented to the network after finishing the network training to examine the network capability in predicting new data. The other 15 data were employed as the training data.

The algorithm was written using MATLAB $\mathrm{R} 2014 \mathrm{~b}$ and run for 5000 iterations. The range of $\mathrm{Sp}$ was selected to be between $[0.1,10]$ with the step of $10^{-2}$. Likewise, $N$ was set to vary between $[1,15]$ with the step of 1 . The convergence plot of the SA algorithm is illustrated in Figure 9.

It can be seen that due to the random nature of the SA, in the early iterations, the objective function values are very large. Furthermore, the behavior of the objective function values is strongly oscillating, and it cannot be confidently concluded that the best answer has been found. The minimum value of the objective

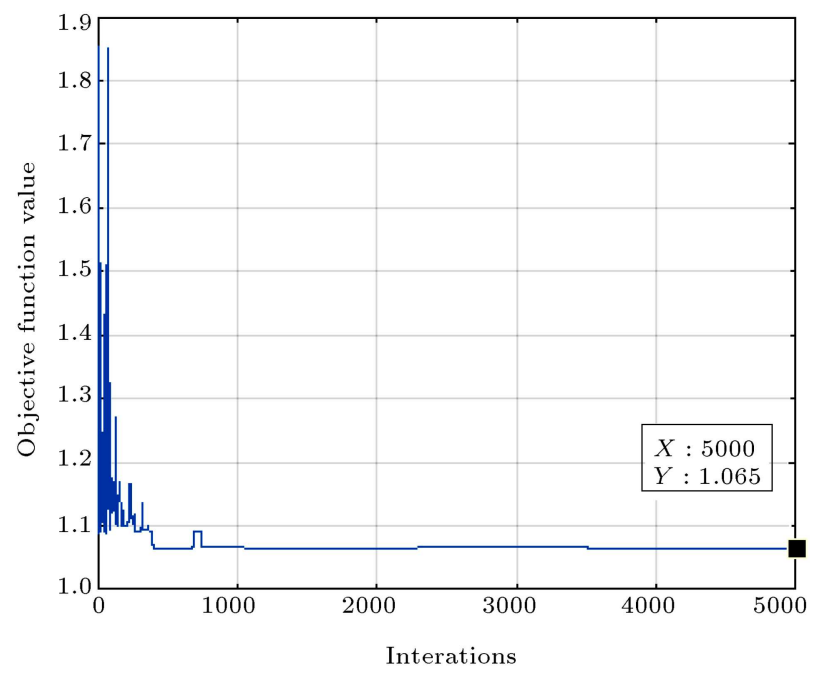

Figure 9. The SA convergence procedure for optimizing the RBF parameters. 
Table 4. The performed experiments (dimensions are in millimeter).

\begin{tabular}{|c|c|c|c|c|}
\hline $\begin{array}{c}\text { Experiment } \\
\text { no. }\end{array}$ & $\begin{array}{c}\text { Position of } \\
\text { locator } 1\end{array}$ & $\begin{array}{c}\text { Position of } \\
\text { locator } 2\end{array}$ & $\begin{array}{c}\text { Position of } \\
\text { locator } 3 \\
\end{array}$ & Distortion \\
\hline 1 & $(0,0)$ & $(0,66)$ & $(0,132)$ & 18.81 \\
\hline 2 & $(0,0)$ & $(41,66)$ & $(41,132)$ & 18.64 \\
\hline 3 & $(0,0)$ & $(82,66)$ & $(82,132)$ & 19.82 \\
\hline 4 & $(0,0)$ & $(123,66)$ & $(123,132)$ & 17.95 \\
\hline 5 & $(41,0)$ & $(0,66)$ & $(41,132)$ & 14.66 \\
\hline 6 & $(41,0)$ & $(41,66)$ & $(0,132)$ & 16.47 \\
\hline 7 & $(41,0)$ & $(82,66)$ & $(123,132)$ & 20.89 \\
\hline 8 & $(41,0)$ & $(123,66)$ & $(82,132)$ & 18.80 \\
\hline 9 & $(82,0)$ & $(0,66)$ & $(82,132)$ & 16.88 \\
\hline 10 & $(82,0)$ & $(41,66)$ & $(123,132)$ & 20.70 \\
\hline 11 & $(82,0)$ & $(82,66)$ & $(0,132)$ & 14.34 \\
\hline 12 & $(82,0)$ & $(123,66)$ & $(41,132)$ & 18.54 \\
\hline 13 & $(123,0)$ & $(0,66)$ & $(123,132)$ & 15.65 \\
\hline 14 & $(123,0)$ & $(41,66)$ & $(82,132)$ & 16.33 \\
\hline 15 & $(123,0)$ & $(82,66)$ & $(41,132)$ & 21.23 \\
\hline 16 & $(123,0)$ & $(123,66)$ & $(0,132)$ & 17.25 \\
\hline 17 & $(123,0)$ & $(0,66)$ & $(82,132)$ & 17.16 \\
\hline 18 & $(82,0)$ & $(0,66)$ & $(41,132)$ & 16.09 \\
\hline 19 & $(82,0)$ & $(41,66)$ & $(82,132)$ & 18.71 \\
\hline 20 & $(0,0)$ & $(123,66)$ & $(0,132)$ & 16.37 \\
\hline 21 & $(82,0)$ & $(0,66)$ & $(0,132)$ & 17.06 \\
\hline
\end{tabular}

function is 1.065 and it occurs at $\vec{X}=(0.86,9)$. By considering the computational cost, the number of iterations is set to 5000. It is clear that approximately after 3500 iterations, no improvement can be observed in the objective function value; but, to ensure that the algorithm will converge on the optimum value, more iterations are needed. To better realize the behavior of the objective function, it is noteworthy to check the standard deviation of the objective function for every 200 iterations (see Figure 10). The function shown in Figure 10 is a decreasing function. It can be seen that, in the initial iterations, the standard deviations are high. When the number of iterations increases, the standard deviation of the objective function decreases. In the final iterations, the standard deviation of the objective function approximately tends to zero. This is because the algorithm converges on the optimum point.

The optimal parameters obtained from the SA lead to finding the best outcomes for the radial basis function network. Using the attained RBF parameters, the network outputs against the targets and the correlation plot for the train data are presented in Figures 11 and 12 , respectively. The figures show that the training procedure has successfully been performed.

Now, to examine the performance of the network against the data not used in the training, the test dataset is presented to the trained network.

The relative error, which is hereafter briefly called

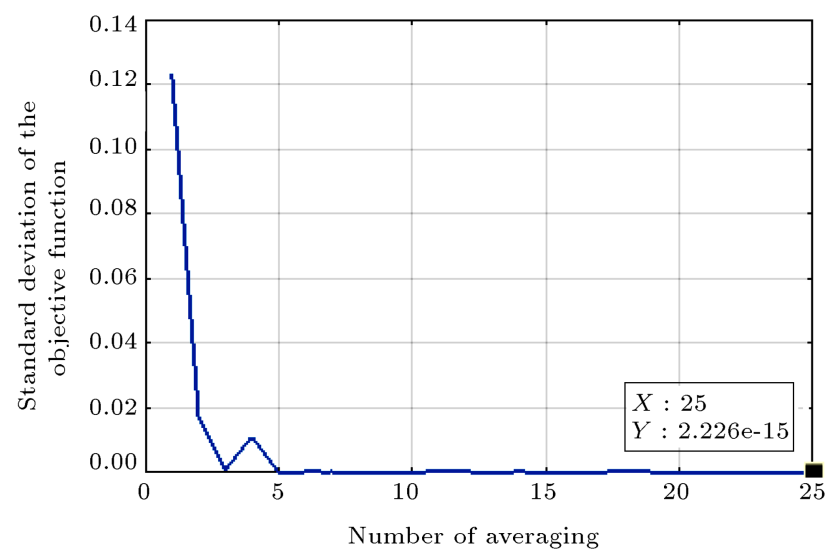

Figure 10. The standard deviation of the objective function for every 200 iterations. 


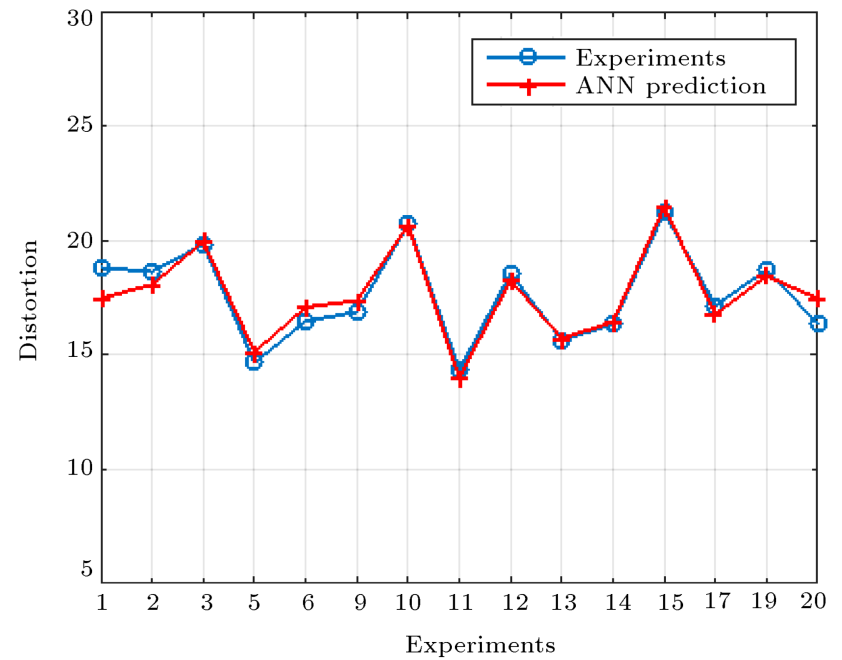

Figure 11. Predicted outputs versus the targets for the train dataset.

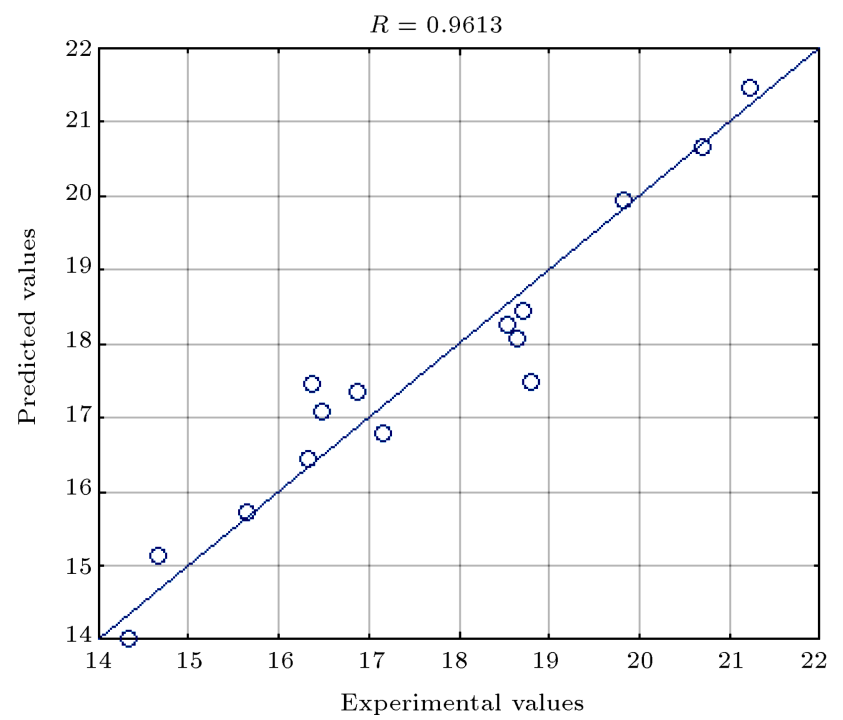

Figure 12. The correlation between the predicted values by the RBF and the targets for the train data.

the error, is a suitable criterion to evaluate the performance of an ANN. It is computed via Eq. (8). In Eq. (8), $T$ and $O$ are respectively the targets and the network outputs.

$$
R E=\frac{|T-O|}{T} \times 100 .
$$

The network outputs versus the targets and the plot of correlation between the targets and the network output have been illustrated in Figures 13 and 14, respectively. The figures prove that the targets and the predicted values are very close and the correlation between both datasets is high.

Furthermore, the target values, the network outputs, and errors calculated for each of the test data have been displayed collectively in Figure 15. It can

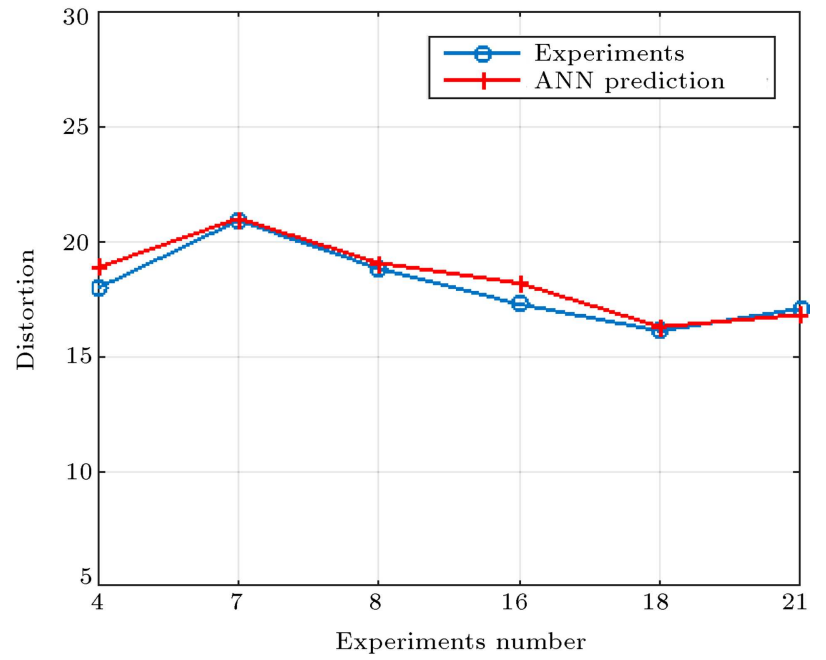

Figure 13. Predicted outputs versus the targets for the test dataset.

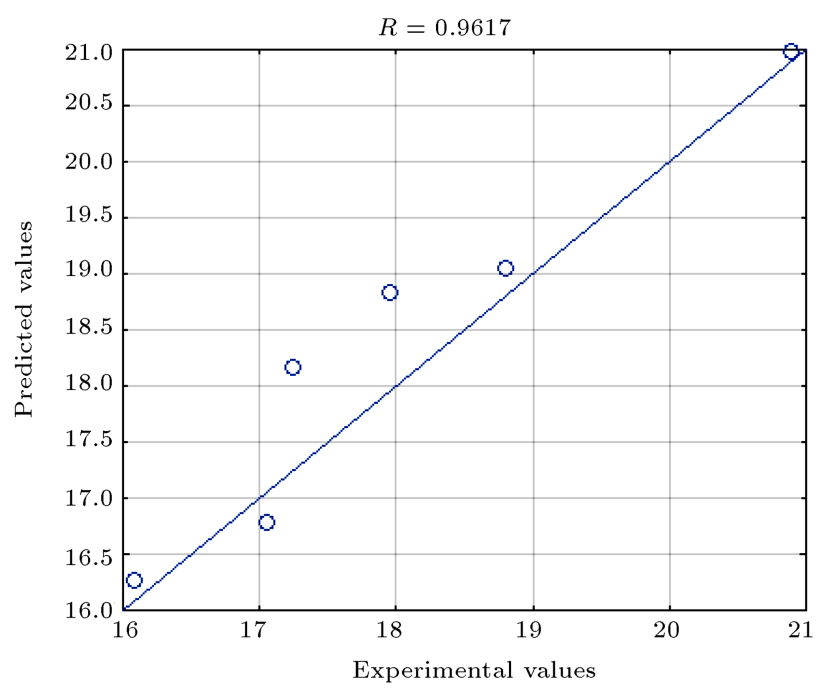

Figure 14. The correlation between the predicted values by the RBF and the targets for the test data.

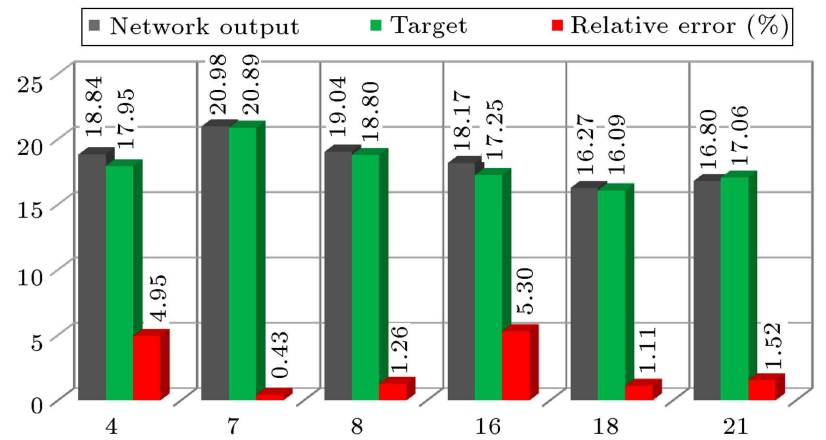

Figure 15. The errors calculated for the test data together with the targets and network outputs.

be seen that the relative errors are all acceptable. Especially, the maximum error (5.30\%) corresponds to datum number 16 and the minimum one $(0.43 \%)$ corresponds to datum number 7 . 
As it is shown, the optimized radial basis function has a high capability in predicting the welding induced distortion. Since the welding distortions have a high degree of nonlinearity and the process of preparing welding setup and test samples is very expensive and time consuming, proposing such an approach that can accurately model the process is very crucial.

\section{Conclusion}

Welding induced distortion is a very complicated and nonlinear phenomenon. Also, purely experimental approach to measure distortions under various settings is very time consuming and expensive, and it needs skillful operators. In this research, it was shown that the RBF was a powerful tool for modeling the GTAW process and predicting the resultant distortions using a limited number of experimental data. In this way, an accurate distortion prediction could be achieved under a given arrangement of the locators in 3-2-1 locating method. The experiments were conducted on the St12 steel plates widely used in automotive industry and other consumer products. To apply the 3-2-1 locating scheme to the sheet metals, a suitable fixture was designed.

In the modeling process, the performance of the RBF model was further improved using the SA algorithm. The RBF outputs obtained for both the training and test datasets were very close to the actual values. The average error computed for the test dataset was $2.43 \%$ with maximum error value of $5.30 \%$ and the minimum value less than $1 \%$. Furthermore, the correlation coefficient between the targets and the outputs of the network when the test data were presented to the network was 0.9617 . This shows that both sets of the data were highly correlated.

These findings illustrate that the proposed approach can effectively be employed to evaluate any given arrangement of the locators in terms of the outof-plane distortions. In this way, the best possible arrangement of the locators may be identified and implemented. This part is an ongoing research.

\section{References}

1. Ates, H., Dursun, B., and Kurt, E. "Estimation of mechanical properties of welded S355J2+ N steel via the artificial neural network", Scientia Iranica Transaction B: Mechanical Engineering, 23(2), p. 609 (2016).

2. Zhang, W.W., Cong, S., Huang, Y., and Tian, Y.H. "Micro structure and mechanical properties of vacuum brazed martensitic stainless steel/tin bronze by Agbased alloy", Journal of Materials Processing Technology, 248 (Supplement C), pp. 64-71 (2017).
3. Narang, H., Mahapatra, M., Jha, P., and Biswas, P. "Optimization and prediction of angular distortion and weldment characteristics of TIG square butt joints", Journal of Materials Engineering and Performance, 23(5), pp. 1750-1758 (2014).

4. Ma, N., Wang, J., and Okumoto, Y. "Out-of-plane welding distortion prediction and mitigation in stiffened welded structures", The International Journal of Advanced Manufacturing Technology, 84(5-8), pp. 1371-1389 (2016).

5. Pazooki, A., Hermans, M., and Richardson, I. "Control of welding distortion during gas metal arc welding of AH36 plates by stress engineering", The International Journal of Advanced Manufacturing Technology, 88(58), pp. 1439-1457 (2017).

6. Michaleris, P., Minimization of Welding Distortion and Buckling: Modelling and Implementation, Michaleris, P., Ed., 1st, Edn., pp. 3-4, Woodhead Publishing, Cambridge CB22 3HJ, UK (2011).

7. Deng, D., Liu, X., He, J., and Liang, W. "Investigating the influence of external restraint on welding distortion in thin-plate bead-on joint by means of numerical simulation and experiment", The International Journal of Advanced Manufacturing Technology, 82(5-8), pp. 1049-1062 (2016).

8. Li, Y., Zhao, Y., Li, Q., et al. "Effects of welding condition on weld shape and distortion in electron beam welded Ti2AlNb alloy joints", Materials \& Design, 114, pp. 226-233 (2017).

9. Lightfoot, M., McPherson, N., Woods, K., and Bruce, G.D. "Artificial neural networks as an aid to steel plate distortion reduction", Journal of Materials Processing Technology, 172(2), pp. 238-242 (2006).

10. Bruce, G. and Lightfoot, M. "The use of artificial neural networks to model distortion caused by welding", International Journal of Modelling and Simulation, 27(1), pp. 32-37 (2007).

11. Yang, H. and Shao, H. "Distortion-oriented welding path optimization based on elastic net method and genetic algorithm", Journal of Materials Processing Technology, 209(9), pp. 4407-4412 (2009).

12. Choobi, M.S., Haghpanahi, M., and Sedighi, M. "Prediction of welding-induced angular distortions in thin butt-welded plates using artificial neural networks", Computational Materials Science, 62, pp. 152-159 (2012).

13. Barclay, C., Campbell, S., Galloway, A., and McPherson, N. "Artificial neural network prediction of weld distortion rectification using a travelling induction coil", The International Journal of Advanced Manufacturing Technology, 68(1-4), pp. 127-140 (2013).

14. Tian, L., Luo, Y., Wang, Y., and Wu, X. "Prediction of transverse and angular distortions of gas tungsten arc bead-on-plate welding using artificial neural network", Materials \& Design (1980-2015), 54, pp. 458-472 (2014). 
15. Narayanareddy, V., Chandrasekhar, N., Vasudevan, M., Muthukumaran, S., and Vasantharaja, P. "Numerical simulation and artificial neural network modeling for predicting welding-induced distortion in buttwelded 304L stainless steel plates", Metallurgical and Materials Transactions B, 47(1), pp. 702-713 (2016).

16. Ma, N., Huang, H., and Murakawa, H. "Effect of jig constraint position and pitch on welding deformation", Journal of Materials Processing Technology, 221, pp. 154-162 (2015).

17. Choobi, M.S., Haghpanahi, M., and Sedighi, M. "Investigation of the effect of clamping on residual stresses and distortions in butt-welded plates", Scientia Iranica Transaction B: Mechanical Engineering, 17(5), p. 387 (2010).

18. Ma, N. and Huang, H. "Efficient simulation of welding distortion in large structures and its reduction by jig constraints", Journal of Materials Engineering and Performance, 26(11), pp. 5206-5216 (2017).

19. Lorin, S., Cromvik, C., Edelvik, F., Lindkvist, L., and Söderberg, R. "Variation simulation of welded assemblies using a thermo-elastic finite element model", Journal of Computing and Information Science in Engineering, 14(3), p. 031003 (2014).

20. Cai, W., Hu, S.J., and Yuan, J. "Deformable sheet metal fixturing: principles, algorithms, and simulations", Journal of Manufacturing Science and Engineering, 118(3), pp. 318-324 (1996).

21. Liu, G., Jia, L., Kong, B., Guan, K., and Zhang, H. "Artificial neural network application to study quantitative relationship between silicide and fracture toughness of Nb-Si alloys", Materials \& Design, 129, pp. 210-218 (2017).

22. Santos, R.B., Ruppb, M., Bonzi, S.J., and Filetia, A.M.F. "Comparison between multilayer feed forward neural networks and a radial basis function network to detect and locate leaks in pipelines transporting gas", Chem. Eng. Trans, 32(1375), p. e1380 (2013).

23. Yu, H., Xie, T., Paszczynski, S., and Wilamowski, B.M. "Advantages of radial basis function networks for dynamic system design", IEEE Transactions on Industrial Electronics, 58(12), pp. 5438-5450 (2011).

24. Azadi Moghaddam, M., Golmezerji, R., and Kolahan, F. "Simultaneous optimization of joint edge geometry and process parameters in gas metal arc welding using integrated ANN-PSO approach", Scientia Iranica Transaction B: Mechanical Engineering, 24(1), pp. 260-273 (2017).

25. Kirkpatrick, S., Gelatt, C.D., and Vecchi, M.P. "Optimization by simulated annealing", science, 220(4598), pp. 671-680 (1983).

\section{Biographies}

Mohammad Mahdi Tafarroj was born on August 2, 1984, in Behbahan, Khouzestan, Iran. He is currently a $\mathrm{PhD}$ student of Mechanical Engineering in the Department of Mechanical Engineering at Ferdowsi University of Mashhad. He received his BSc degree in Mechanical Engineering from Shahid Chamran University of Ahvaz, Iran, in 2008 and MSc from Islamic Azad University of Ahvaz in 2011. His main research interests are welding, artificial neural networks, optimization, fuzzy logic, signal processing, data mining, image processing, and fault detection and diagnosis.

Farhad Kolahan was born in September 1965 in Mashhad, Iran. He is currently an Associate Professor in the Department of Mechanical Engineering at Ferdowsi University of Mashhad, Iran. He received his BSc degree in Production and Manufacturing Engineering from Tabriz University, Tabriz, Iran. Then, he continued his postgraduate studies abroad and was graduated with a $\mathrm{PhD}$ degree in Industrial and Manufacturing Engineering from Ottawa University, Canada, in 1999. Dr. Kolahan's research interests include welding, production planning and scheduling, manufacturing processes optimization, and applications of heuristic algorithms in industrial optimization. 